\title{
A Joint Channel Estimation and Data Detection for a MIMO Wireless Communication System via Sphere Decoding
}

\author{
Gajanan R. Patil ${ }^{\star}$ and Vishwanath K. Kokate**
}

\begin{abstract}
A joint channel estimation and data detection technique for a multiple input multiple output (MIMO) wireless communication system is proposed. It combines the least square (LS) training based channel estimation (TBCE) scheme with sphere decoding. In this new approach, channel estimation is enhanced with the help of blind symbols, which are selected based on their correctness. The correctness is determined via sphere decoding. The performance of the new scheme is studied through simulation in terms of the bit error rate (BER). The results show that the proposed channel estimation has comparable performance and better computational complexity over the existing semi-blind channel estimation (SBCE) method.
\end{abstract}

\section{Keywords}

Maximum Likelihood Decoding, Multiple Input Multiple Output, Normalized Mean Square Error, Training Based Channel Estimation, Semi-Blind Channel Estimation, Spatial Multiplexing, Sphere Decoding

\section{Introduction}

The demand for high data rate multimedia systems in a wireless scenario is a major driving force for research in the area of multiple input multiple output (MIMO) wireless communication systems. It has been shown that the use of multiple antennas at the transmitter and or the receiver can improve the capacity of the communication system [1-3]. The simple transmit diversity scheme suggested by Alamouti [4] and the space time coding suggested by Tarokh et al. [5] triggered research in this area. Several transmission schemes that utilize the MIMO channel in different ways have been proposed. These schemes can be categorized as spatial multiplexing, spatial diversity (space-time coding), smart antennas, and beam forming techniques [6].

The system's ability to achieve MIMO capacity depends on channel state information. Accurately estimating the MIMO channel is much more challenging than doing for the SISO channel [7]. There are a number of channel estimation schemes suggested in the literature on channel estimation for MIMO systems. These schemes can be categorized as training-based channel estimation (TBCE), blind channel estimation (BCE) and semi-blind channel estimation (SBCE). Training based schemes are capable of accurately estimating a MIMO channel, provided a large training overhead is made available. Hence,

\footnotetext{
* This is an Open Access article distributed under the terms of the Creative Commons Attribution Non-Commercial License (http://creativecommons. org/licenses/by-nc/3.0/) which permits unrestricted non-commercial use, distribution, and reproduction in any medium, provided the original work is properly cited.

Manuscript received March 14, 2014; accepted May 14, 2014; onlinefirst July 31, 2015.

Corresponding Author: Gajanan R. Patil (patilgr67@yahoo.co.in)

* Dept. of Electronics and Telecommunication Engineering, Army Institute of Technology, Pune, India (patilgr67@yahoo.co.in)

** Dept. of Electronics and Telecommunication Engineering, Sinhgad College of Engineering, Pune, India (vkkokateetc@gmail.com)
} 
there is a considerable reduction in system throughput. The least square (LS) and minimum mean square error (MMSE) techniques are widely used for channel estimation when training symbols are available. The LS method is simpler than MMSE but the performance of the MMSE scheme is better. However, the MMSE method requires knowledge about channel correlation. Blind methods do not require the training overhead. These methods not only impose high complexity and slow convergence, but also suffer from unavoidable estimation and decision ambiguities. Semi-blind methods offer attractive practical means of implementing MIMO systems. SBCE schemes use a few training symbols to provide the initial MIMO channel estimation and make use of blind information to further improve the estimation. Some SBCE schemes also exchange the information between the channel estimator and the data detector iteratively.

Several SBCE solutions have been proposed to minimize the computational cost, and hence, the energy spent in channel estimation of MIMO systems. The SBCE schemes suggested in [8,9] use few training symbols to provide an initial estimate and then the data detector and estimator exchange the information iteratively. In [10-12], the MIMO channel matrix is decomposed into whitening and the rotation matrix. The whitening matrix is estimated using blind symbols and the rotation matrix is estimated using few pilot symbols. The OPML estimator shows a 1-dB improvement of bit error rate (BER) compared to the conventional LS training scheme if the same length of training sequence is used. Furthermore, SVD has to be applied twice to obtain the whitening matrix and the rotation matrix. These operations lead to increased computational complexity [13]. In [14], TBCE and SBCE, considering Perfect, LS, LMMSE, ML, and MAP estimators, are studied in terms of BER and complexity. Subspace based SBCE is discussed in $[15,16]$. A linear prediction based semi-blind estimation for the FIR MIMO channel is proposed in [17]. A decision directed iterative channel estimation proposed in [18] improves the computational complexity by avoiding matrix inversion. The number of semi-blind channel estimation schemes are reported for MIMO-OFDM systems as well [19-26].

Spatial multiplexing can be used in MIMO systems to achieve a higher transmission rate without allocating a higher bandwidth or increasing transmission power. At the receiver side, the main challenge resides in designing powerful signal processing techniques (i.e., detection techniques) that are capable of separating the transmitted signals. Different research activities have been carried out to show that the spatial multiplexing concept has the potential to significantly increase spectral efficiency [27]. In general, these techniques assume channel knowledge at the receiver and the performance can be further improved when the knowledge of the channel response is available at the transmitter. However, spatial multiplexing does not work well in low SNR environments, as it is more difficult for the receiver to recognize the multiple uncorrelated paths of the signals. The main challenge in the practical realization of MIMO wireless systems lies in the efficient implementation of the detector, which needs to separate the spatially multiplexed data streams. So far, several algorithms offering various tradeoffs between performance and computational complexity have been developed [27]. Several MIMO detection techniques were proposed in the literature on MIMO systems. MIMO detection techniques are categorized as maximum likelihood detectors (MLDs), linear detectors, successive interference cancelation, and tree-search techniques. The MLD has an optimum performance but the complexity of the algorithm increases exponentially with the number of transmitting antennas and the order of modulation, which makes its implementation feasible [28]. The sphere decoding algorithm is used to reduce the computational complexity of MLD. Several sphere decoding algorithms and their implementation aspects are discussed in the literature on MIMO systems [29-31]. A radius selection algorithm for a sphere decoder is proposed in [32]. The authors of the 
paper in [33] have studied the simple implementation of the sphere decoding algorithm and its performance for MIMO and MIMO-OFDM systems using it. The authors in [34] studied the performance of spatial multiplexing systems with MLD.

This paper proposes a new approach to joint channel estimation and data detection in which the channel is estimated with few training symbols and estimation is enhanced with the help of data detection iteratively. The data detection is carried out via sphere decoding. The performance of the channel estimation is improved with the help of more reliable blind data, which can be identified through sphere decoding technique.

The rest of this paper is organized as follows: Section 2 describes the system model. The design of the proposed estimator is given in Section 3. In Section 4, we share and discuss the simulation results. Finally, the conclusion is given in Section 5.

Throughout our discussions we adopt the following notational conventions. Boldface capital letter and lower-case letters stand for matrices and vectors, respectively. We denoted the identity matrix. $(.)^{\mathrm{H}}$ and $(.)^{\dagger}$ are the conjugate transpose and the Moore-Penrose pseudo-inverse operators, respectively; while $\|.\|_{\mathrm{F}}^{2}$ and $|$.$| denote the Frobenius norm and magnitude operators, respectively. Finally, E($.$) is$ the expectation operator.

\section{System Model}

Consider a spatial multiplexing based MIMO system shown in Fig. 1 with the $\mathrm{M}$ transmit and $\mathrm{N}$ receives antennas where, $N \geq M$.

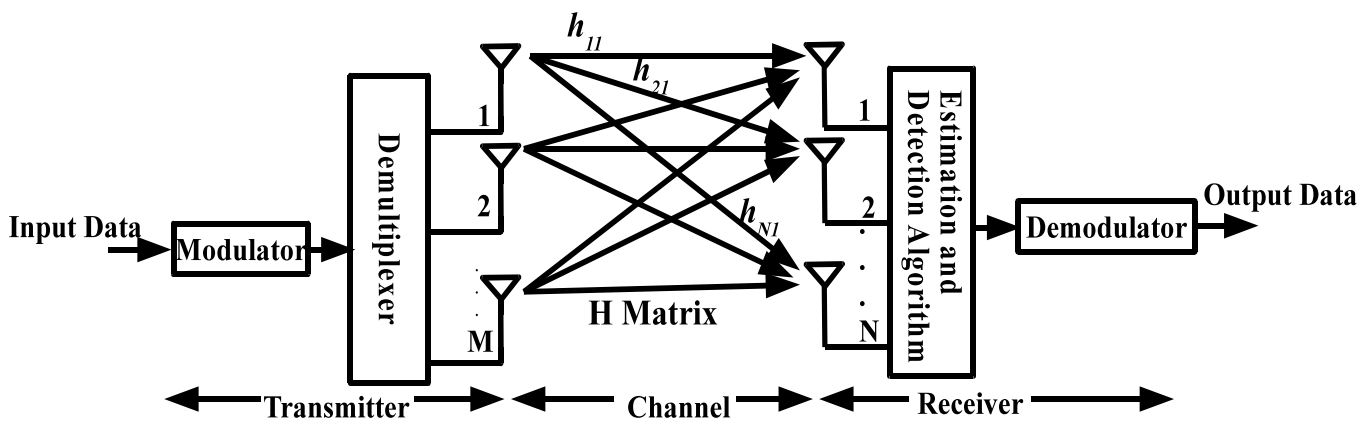

Fig. 1. $M \times N$ MIMO system with spatial multiplexing.

Let us assume the channel to be Rayleigh flat fading with channel matrix $\boldsymbol{H} \in \mathbb{C}^{N \times M}$. Each element $h_{i j}$ in the matrix represents the flat fading channel coefficient between the $i$-th receiver and the $j$-th transmitter antenna. Independent data streams are modulated and transmitted on $M$ different antennas. Denoting the complex received data by $\boldsymbol{Y} \in \mathbb{C}^{N \times 1}$ the equivalent baseband system can be modeled as [10]:

$$
\boldsymbol{Y}=\boldsymbol{H} \boldsymbol{X}+\boldsymbol{n}
$$

where, $\boldsymbol{X} \in \mathbb{C}^{M \times 1}$ is the transmitted symbol vector and $\boldsymbol{n}$ is the additive white Gaussian noise with zero mean and noise power $\sigma_{n}^{2}$. Also, the sources are assumed to be spatially and temporally independent 
with identical source power $\sigma_{s}^{2}$. The system model of Eq. (1) can be given in matrix form as:

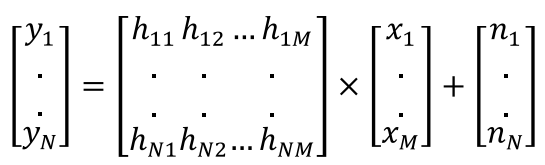

Assume that the channel remains constant for $K$ symbol periods. In these $K$ symbols, the first $L$ symbols are used for training. Let these training symbol vectors be $\boldsymbol{X}_{\boldsymbol{p}}=\left[\mathbf{x}_{1}, \mathbf{x}_{2}, \mathbf{x}_{3} \ldots, \mathbf{x}_{\mathrm{L}}\right]$ where $\boldsymbol{X}_{\boldsymbol{p}} \in \mathbb{C}^{M \times L}$ and its corresponding output $\boldsymbol{Y}_{\boldsymbol{p}} \in \mathbb{C}^{N \times L}$ is the received training symbol output vectors. The remaining $K-L$ symbol vectors are blind data symbols $\boldsymbol{X}_{\boldsymbol{b}}$ and their corresponding output $\boldsymbol{Y}_{\boldsymbol{b}}$ where $\boldsymbol{X}_{\boldsymbol{b}} \in \mathbb{C}^{M \times K-L}$ and $\boldsymbol{Y}_{\boldsymbol{b}} \in \mathbb{C}^{N \times K-L}$.

\section{Proposed Algorithm for Joint Estimation and Detection}

\subsection{Training Based Channel Estimation}

The matrix $\boldsymbol{H}$ can be estimated using only training symbols using LS estimation [35]. The LS estimate of the channel $\widehat{\boldsymbol{H}}_{\boldsymbol{l}}$, can be obtained by minimizing $\left\|\boldsymbol{H} \boldsymbol{X}_{\boldsymbol{p}}-\boldsymbol{Y}_{\boldsymbol{p}}\right\|^{2}$. Then the LS estimation of $\boldsymbol{H}$ is given by Eq. (2)

$$
\widehat{H}_{l s}=\boldsymbol{Y}_{p} \boldsymbol{X}_{p}^{\dagger}
$$

where, $\boldsymbol{X}_{\boldsymbol{p}}^{\dagger}$ denotes the Moore-Penrose pseudo-inverse of $\boldsymbol{X}_{\boldsymbol{p}}$.

Training based channel estimation techniques are easy to implement, but for more accurate channel estimation we require more training symbols. However, redundant training information affects the spectral efficiency.

\subsection{Joint Channel Estimation and Data Detection}

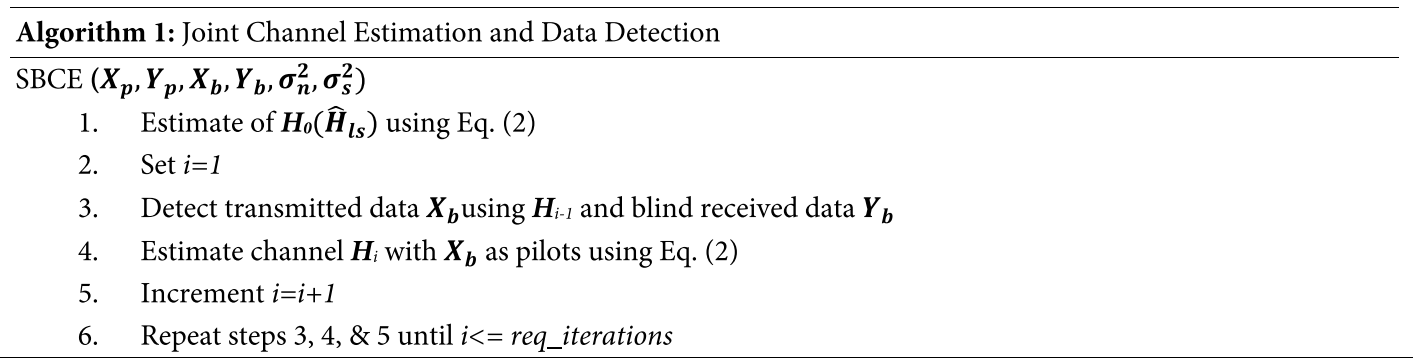

The efficient usage of bandwidth can be achieved through blind methods, but they have a poor convergence rate and require long data records to be processed. Hence, techniques that use a small amount of training symbols with blind data can provide an attractive solution for channel estimation. In joint estimation and data detection, the data decision obtained from the decoding, either hard or soft, is used as additional training to refine the channel estimate [14] and [18]. Algorithm 1 gives the procedure for joint channel estimation and data detection.

The above algorithm has the following drawbacks: 
i) It assumes that all of the detected symbols to be correctly detected, which is dependent on the number of errors in the transmission. Thus, at low SNR these errors are more and the estimate will be less accurate. At high SNR these errors are relatively lower and the estimate will be more accurate and better.

ii) The computational complexity increases as the pseudo-inverse of a large size matrix is to be obtained in every step.

iii) The MLD is computationally complex.

The new joint channel estimation and data detection is proposed for estimating the MIMO channel, which eliminates these drawbacks. The sphere decoding technique is used instead of MLD. The computational complexity of sphere decoding is better than MLD and it gives near optimal performance. Instead of considering all of the detected symbols, only a few accurately detected symbols are used for channel estimation so that the inverse of a large size matrix is avoided. The accurately detected symbols can be obtained from the sphere decoder.

\subsection{Sphere Decoder}

Assuming $\boldsymbol{H}$ is known at the receiver, the estimated symbol stream using MLD is given by:

$$
\tilde{\boldsymbol{x}}=\arg \min _{\boldsymbol{x}_{\boldsymbol{k}} \in\left\{x_{1}, x_{2} \ldots x_{M}\right\}}\left\|\boldsymbol{r}-\boldsymbol{H} \boldsymbol{x}_{\boldsymbol{k}}\right\|^{2}
$$

Using the above criterion, MLD compares the received signal with the entire possible transmitted symbol streams, which have undergone the Rayleigh fading. The sphere decoder (SD) algorithm consists of two stages viz. the pre-processing stage and searching stage. Pre-processing such as QR decomposition and lattice reduction transforms the problem so that the complexity of the problem is reduced. The searching stage finds the closest point in the hyper sphere [27].

The SD algorithms can solve the ML detection problem in Eq. (2) by searching over a restricted subset $\Omega$ that at least contains the ML solution. The channel matrix $H$ is decomposed into $H=Q R$, where $\boldsymbol{R}$ is the upper triangular and $\boldsymbol{Q}$ has orthogonal columns with unit norms. Hence, the system model in Eq. (1) can be written as,

$$
\boldsymbol{y}=\boldsymbol{R} x+v
$$

Where $\boldsymbol{y}=\boldsymbol{Q}^{H} \boldsymbol{r}$ and $\boldsymbol{v}=\boldsymbol{Q}^{H} \boldsymbol{n}$ and the ML problem in Eq. (2) can be written as:

$$
\tilde{x}=\arg \min _{\boldsymbol{x}_{\boldsymbol{k}} \in \Omega}\left\|\boldsymbol{y}-\boldsymbol{R} \boldsymbol{x}_{\boldsymbol{k}}\right\|^{2}
$$

The sphere decoder solves Eq. (5) satisfying the constraint:

$$
\left\|\boldsymbol{y}-\boldsymbol{R} \boldsymbol{x}_{\boldsymbol{k}}\right\|^{2} \leq \text { radius }^{2}
$$

The SD algorithm for the solving Eq. (5) can be implemented through a depth first search (DFS) tree traversal using Eq. (6) as pruning criteria to reduce the search. The node at the $k$-th level represents a 
sequence of symbols from $x_{M-k+1}$ to $x_{M}$ counting from the root of the tree, which is at the 0th level. All of the leaf nodes in the tree will represent the full sequence $x_{1}$ to $x_{M}$. The algorithm searches all of the nodes in the tree that will satisfy constraint given in Eq. (6). The initial choice of the radius decides the performance of the sphere decoding algorithm. Instead of going through every point in the hyper sphere the adaptive sphere decoding algorithm remembers the currently closest point and compares it with a new point to determine whether the new point is closer than the known one. This algorithm keeps on updating the sphere radius to avoid searching the unnecessary lattice points.

The final radius with which we make a decoding decision can give us information about how accurate our detection is. The smaller the radius the more accurate our decision can be. Algorithm 1 uses all of the blind symbols irrespective of whether the symbols are detected correctly or not to estimate the channel. This paper proposes a new approach to improve the computational complexity without sacrificing the performance. We can use a fewer number of blind symbols, which are more accurately detected. Hence, we can select symbols detected with lower radius. Algorithm 2 illustrates this. Since we are using few training symbols and blind data for channel estimation it is a SBCE approach.

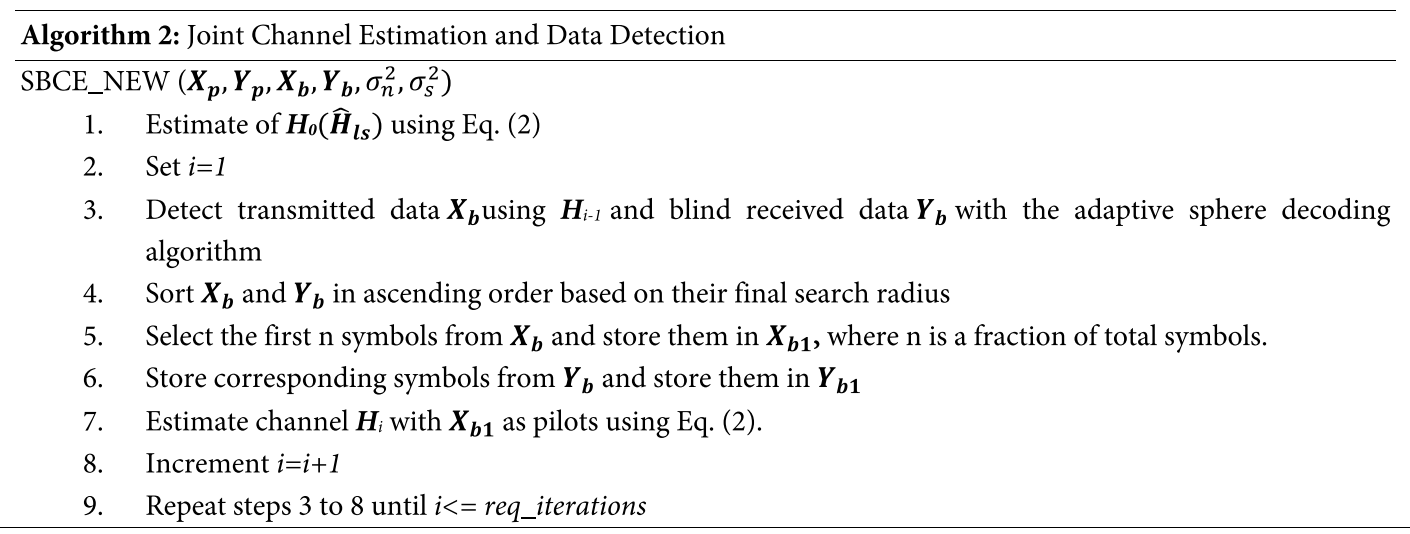

Since we are using very few detected symbols as pilots, the computational complexity of the algorithm is better than Algorithm 1. For the low SNR, more reliable detected symbols are used for channel estimation; hence, the error performance will be better.

\section{Results}

Extensive simulations were carried out in MATLAB 7.8 to test the performance of the proposed channel estimator and they were compared with the estimator of [14]. The simulation was carried out for $2 \times 4$ MIMO systems (two transmitting and four receiving antennas) under the flat fading channel. The spatial multiplexing MIMO system with M-QAM modulation is used for this study. The sphere decoder with an adaptive radius search is used for the detection of symbols

Experiment 1: First we tested the BER performance of the $2 \times 4$ MIMO system using Algorithm 1. In simulation scenarios, 4-QAM data modulation is used with the Rayleigh flat fading MIMO channel. In the simulation, a transmitted frame consists of four orthogonal pilots $(L)$ and 100 blind symbols $(K-L)$. The BER is calculated by averaging over 100 rounds of simulation for different values of Eb/No. Fig. 2 shows 
the BER performance with this setup. The PCE plot shows BER performance with a perfect channel. The TBCE plot shows the BER performance with channel estimation that was obtained using training symbols alone. The SBCE 1 iteration plot shows BER performance with the channel estimate that was obtained using training and all blind symbols. In this case, the channel estimate from training symbol is used to detect blind data. Using this data as pilots, channel estimation is enhanced. In the SBCE 4 iteration plot, the procedure of blind data detection and channel estimation is repeated four times.

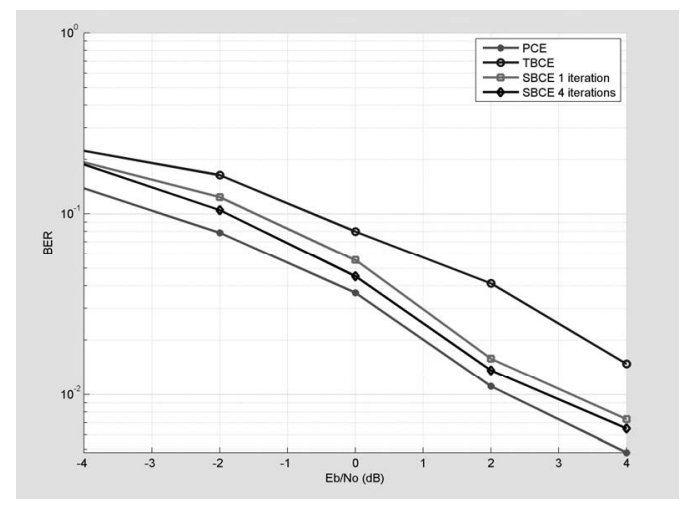

Fig. 2. BER performance of $2 \times 4$ MIMO system with joint channel estimation and data detection (SBCE) for Algorithm 1. BER=bit error rate, $\mathrm{MIMO}=$ multiple input multiple output, $\mathrm{PCE}=$ perfect channel estimation, $\mathrm{SBCE}=$ semi-blind channel estimation, $\mathrm{TBCE}=$ training based channel estimation.

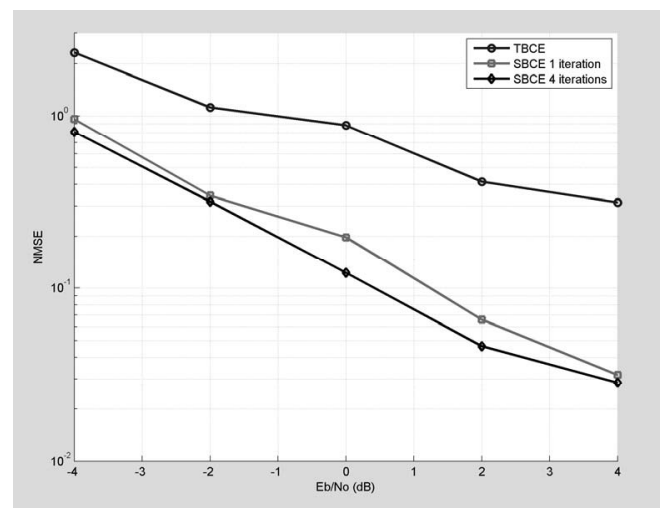

Fig. 3. Normalized mean square error (NMSE) for $2 \times 4$ MIMO system with joint channel estimation and data detection $(\mathrm{SBCE})$ for Algorithm 1. MIMO=multiple input multiple output, $\mathrm{PCE}=$ perfect channel estimation, $\mathrm{SBCE}=$ semi-blind channel estimation, $\mathrm{TBCE}=$ training based channel estimation.

Fig. 3 shows the normalized mean square error (NMSE) for various channel estimations for the same simulation setup, as described above.

The results shown in Figs. 2 and 3 depict that the joint channel estimation and data detection approach of Algorithm 1 has very good performance in terms of BER and NMSE. The performance improves as the number of iterations is increased. But the computational complexity of this approach is very high as the inverse of a large matrix is to be obtained and high complexity detection is required in each iteration. 
Experiment 2: In this experiment, we tested the BER performance of the $2 \times 4$ MIMO system using Algorithm 2. In simulation scenarios, 4-QAM data modulation is used with Rayleigh flat fading MIMO channel. In the simulation, a transmitted frame consists of four orthogonal pilots $(L)$ and 100 blind symbols $(K-L)$. The BER is calculated by averaging over 100 rounds of simulation for different values of $\mathrm{Eb} / \mathrm{No}$. For a given Eb/No value, the initial estimation is obtained using pilots. Then, $40 \%$ of the detected symbols (i.e., 40 out of 100 blind symbols) with the lower final search radius are used for obtaining channel estimation in the subsequent iterations. The performance is compared with channel estimation that is obtained by using entire blind data. The experiment is repeated for $50 \%, 60 \%$, and $80 \%$ of the detected symbols with a lower final search radius.

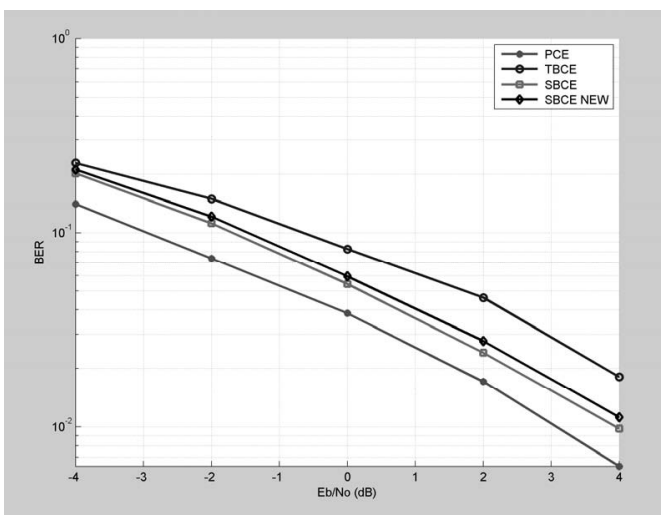

Fig. 4. BER performance of $2 \times 4$ MIMO system with PCE, TBCE and joint channel estimation and data detection with $100 \%$ and $40 \%$ detected symbols (Algorithm 2). BER=bit error rate, MIMO=multiple input multiple output, $\mathrm{PCE}=$ perfect channel estimation, $\mathrm{SBCE}=$ semi-blind channel estimation, $\mathrm{TBCE}=$ training based channel estimation.

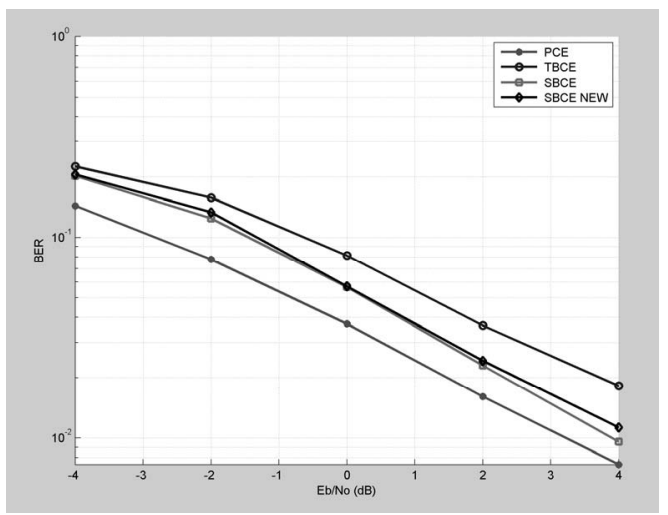

Fig. 5. BER performance of $2 \times 4$ MIMO system with PCE, TBCE and joint channel estimation and data detection with $100 \%$ and $50 \%$ detected symbols (Algorithm 2). BER=bit error rate, MIMO=multiple input multiple output, $\mathrm{PCE}=$ perfect channel estimation, $\mathrm{SBCE}=$ semi-blind channel estimation, $\mathrm{TBCE}=$ training based channel estimation.

Fig. 4 shows the BER performance of the $2 \times 4$ MIMO system with joint channel estimation and data detection where $40 \%$ of the detected symbols with lower final search radius are utilized for channel 
estimation. The BER performance of PCE, TBCE, and SBCE with all of the data is also plotted for comparison. It can be seen that there is a slight degradation of performance with the new approach. The channel estimate is obtained with a single iteration.

Fig. 5 shows the BER performance of the $2 \times 4$ MIMO system with joint channel estimation and data detection where $50 \%$ of the detected symbols are utilized for enhancing the channel estimation. The BER performance of PCE, TBCE, and SBCE with all of the data is also plotted for comparison. Figs. 6 and 7 show the BER performance with $60 \%$ and $80 \%$ of the detected symbols with a lower final search radius.

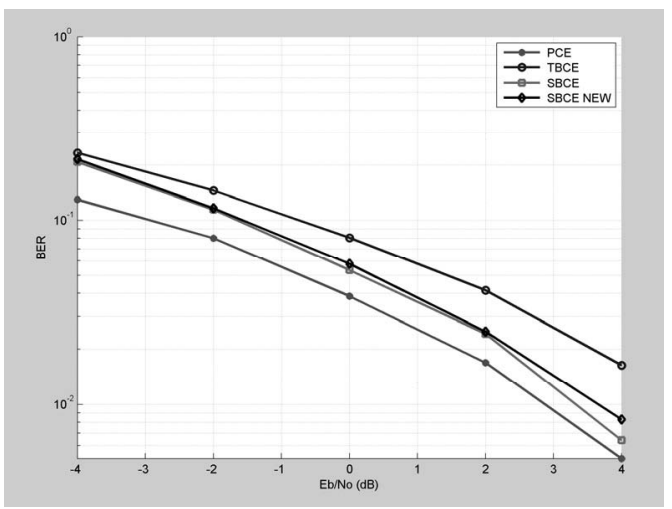

Fig. 6. BER performance of $2 \times 4$ MIMO system with PCE, TBCE and joint channel estimation and data detection with $100 \%$ and $60 \%$ detected symbols (Algorithm 2). BER=bit error rate, MIMO=multiple input multiple output, $\mathrm{PCE}=$ perfect channel estimation, $\mathrm{SBCE}=$ semi-blind channel estimation, $\mathrm{TBCE}=$ training based channel estimation.

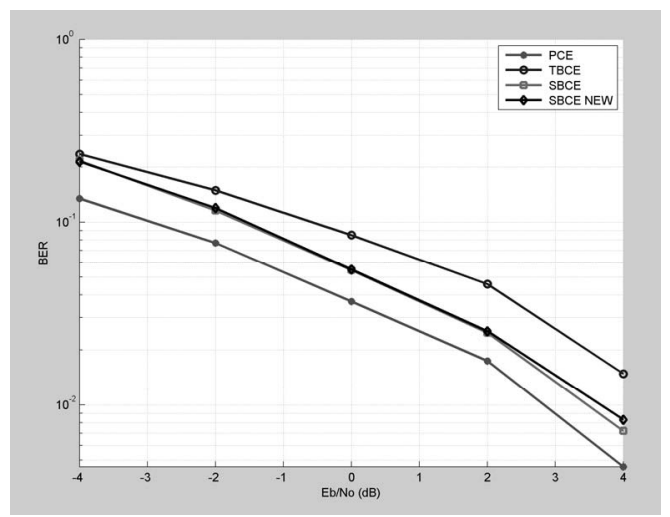

Fig. 7. BER performance of $2 \times 4$ MIMO system with PCE, TBCE and joint channel estimation and data detection with $100 \%$ and $80 \%$ detected symbols (Algorithm 2). BER=bit error rate, MIMO=multiple input multiple output, $\mathrm{PCE}=$ perfect channel estimation, $\mathrm{SBCE}=$ semi-blind channel estimation, $\mathrm{TBCE}=$ training based channel estimation.

The results show that with $50 \%$ of the chosen detected symbols it is possible to estimate the channel. This can result in the same performance as obtained with $100 \%$ of the detected symbols.

The simulations using Algorithm 2 were carried out with single iterations. The performance of the 
$2 \times 4$ MIMO system with Algorithm 2 was also studied with four iterations. Fig. 8 shows the BER performance with $\mathrm{PCE}$, TBCE, joint $\mathrm{CE}$, and data detection using $60 \%$ and $100 \%$ of the detected symbols with a lower final search radius.

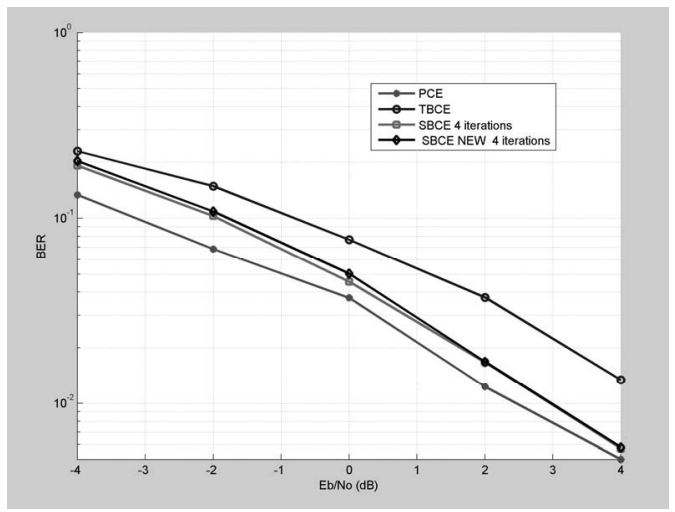

Fig. 8. BER performance of $2 \times 4$ MIMO system with PCE, TBCE and joint channel estimation and data detection for $100 \%$ and $60 \%$ detected symbols using 4 iterations (Algorithm 2). BER=bit error rate, $\mathrm{MIMO}=$ multiple input multiple output, $\mathrm{PCE}=$ perfect channel estimation, $\mathrm{SBCE}=$ semi-blind channel estimation, TBCE=training based channel estimation.

The results obtained indicate that an increase in the number of iterations improves the performance of the system. The channel estimation with $60 \%$ of the detected symbols with a lower final search radius results into almost the same performance as that with $100 \%$ of the detected symbols.

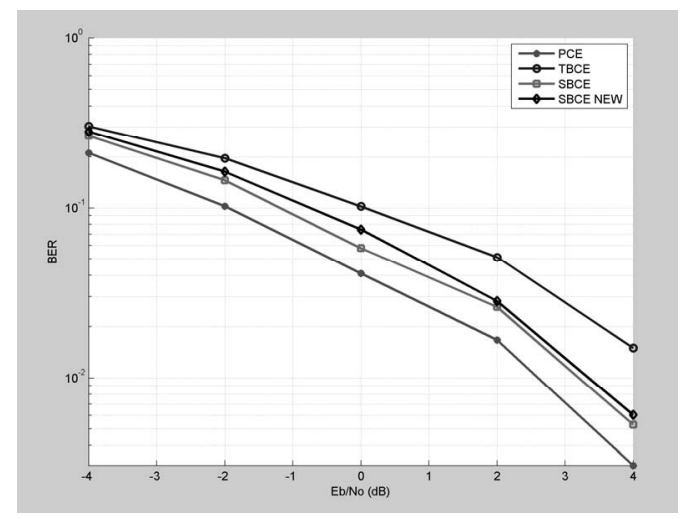

Fig. 9. BER performance of $4 \times 8$ MIMO system with PCE, TBCE and joint channel estimation and data detection for $100 \%$ and $60 \%$ detected symbols using 4 iterations (Algorithm 2). BER=bit error rate, $\mathrm{MIMO}=$ multiple input multiple output, $\mathrm{PCE}=$ perfect channel estimation, $\mathrm{SBCE}=$ semi-blind channel estimation, $\mathrm{TBCE}=$ training based channel estimation.

The BER performance of the $4 \times 8$ MIMO system using Algorithm 2 was also studied. In simulation scenarios, 4-QAM data modulation is used with the Rayleigh flat fading MIMO channel. In the simulation, a transmitted frame consists of eight orthogonal pilots $(L)$ and 200 blind symbols $(K-L)$. The BER is calculated by averaging over 50 rounds of simulation for different values of Eb/No. For a 
given $\mathrm{Eb} /$ No value, initial estimation is obtained using pilots. Then, $60 \%$ of the detected symbols with a lower final search radius are used for obtaining channel estimation in the subsequent iterations. The performance is compared with the channel estimation that was obtained by using all of the blind data along with PCE and TBCE, as plotted. Fig. 9 shows the BER performance for this set up. There is a slight degradation of performance between the estimated channel with $60 \%$ of the detected symbols with a lower final search radius and $100 \%$ of the detected symbols. It was observed that the BER performance of the $2 \times 4$ MIMO and $4 \times 8$ MIMO systems is almost similar. This is because the BER performances are plotted in the low SNR regime where the number of errors will be more in the case of $4 \times 8$ MIMO than that of the $2 \times 4$ MIMO system as compared to a higher SNR regime. But as far as spectral efficiency is concerned, note that we are transmitting double the number of bits in the $4 \times 8$ MIMO system as compared to the $2 \times 4$ MIMO system with the same available bandwidth. For example, if we are transmitting 10,000 bits per second with the $2 \times 4$ MIMO system then accounting for the errors we are transmitting 10,000-1,490 $=8,590$-bits per second with a perfect channel estimator. If we use a $4 \times 8$ MIMO system to transmit the same information, 20,000-bits per second can be transmitted and, taking errors into account, 20,000 -4,080 $=15,920$ bits per second are transmitted effectively. Thus, the capacity of the $4 \times 8 \mathrm{MIMO}$ system is more than the $2 \times 4 \mathrm{MIMO}$ system. If we find the BER performance in a high SNR regime, the $4 \times 8$ MIMO will perform better than the 2 X 4 MIMO provided that perfect channel estimation is available. Table 1 shows the BER performance of the $2 \times 4 \mathrm{MIMO}$ and $4 \times 8 \mathrm{MIMO}$ systems for the proposed algorithm (Algorithm 2) with $60 \%$ of the detected symbols. It can be seen from the table that the performance of the $4 \times 8 \mathrm{MIMO}$ is better than the $2 \times 4 \mathrm{MIMO}$ in terms of BER after $\mathrm{Eb} / \mathrm{No}=6 \mathrm{~dB}$.

Table 1. BER values for $2 \times 4$ and $4 \times 8$ MIMO systems with various estimators

\begin{tabular}{ccccccccc}
\hline Eb/No & \multicolumn{2}{c}{ PCE } & \multicolumn{2}{c}{ TBCE } & \multicolumn{2}{c}{ SBCE } & \multicolumn{2}{c}{ SBCE NEW } \\
\cline { 2 - 9 }$(\mathbf{d B})$ & 2×4 MIMO & 4×8 MIMO & 2×4 MIMO & 4×8 MIMO & 2×4 MIMO & 4×8 MIMO & 2×4 MIMO & 4×8 MIMO \\
\hline-4 & 0.1490 & 0.2040 & 0.2305 & 0.3066 & 0.1888 & 0.2695 & 0.1973 & 0.2913 \\
-2 & 0.0830 & 0.1017 & 0.1533 & 0.1899 & 0.1095 & 0.1390 & 0.1177 & 0.1619 \\
0 & 0.0370 & 0.0469 & 0.0823 & 0.1074 & 0.0447 & 0.0729 & 0.0568 & 0.0901 \\
2 & 0.0133 & 0.0135 & 0.0352 & 0.0440 & 0.0140 & 0.0179 & 0.0153 & 0.0220 \\
4 & 0.0057 & 0.0049 & 0.0145 & 0.0156 & 0.0047 & 0.0064 & 0.0065 & 0.0075 \\
6 & 0.0015 & 0.0009 & 0.0055 & 0.0043 & 0.0015 & 0.0016 & 0.0013 & 0.0018 \\
8 & 0.0008 & 0 & 0.0010 & 0.0003 & 0.0010 & 0 & 0.0008 & 0 \\
\hline
\end{tabular}

$\mathrm{BER}=$ bit error rate, $\mathrm{MIMO}=$ multiple input multiple output, $\mathrm{PCE}=$ perfect channel estimation, $\mathrm{SBCE}=$ semi-blind channel estimation, $\mathrm{TBCE}=$ training based channel estimation.

\section{Conclusion}

A new approach to joint channel estimation and detection for the MIMO wireless communication system has been proposed in this paper. It uses few training symbols for initial estimation and $50 \%-$ $60 \%$ of the blind symbols to further enhance the estimate. The sphere decoding method is utilized to select these blind symbols. Thus, computational complexity is reduced by $40 \%-50 \%$; whereas, the performance is comparable to schemes that utilize $100 \%$ of the symbols. The BER performance of the $2 \times 4$ MIMO and $4 \times 8$ MIMO systems under the Rayleigh flat fading channel has been tested using the 
proposed approach. The channel estimate obtained using the proposed approach is found to be comparable with existing schemes that use few training symbols and $100 \%$ of the blind symbols.

The computational complexity of the proposed method is still higher than the existing semi blind channel estimation techniques. The future work in this area will involve further reduction in computational complexity with a better sphere decoding algorithm. The method can also be extended to MIMO-OFDM systems.

\section{References}

[1] G. J. Foschini, "Layered space-time architecture for wireless communication in a fading environment when using multi-element antennas," Bell Labs Technical Journal, vol. 1, no. 2, pp. 41-59, 1996.

[2] G. J. Foschini and M. J. Gans, "On limits of wireless communications in a fading environment when using multiple antennas," Wireless Personal Communications, vol. 6, no. 3, pp. 311-335, 1998.

[3] I. E. Telatar, “Capacity of multi-antenna Gaussian channels," European Transactions on Telecommunications, vol. 10, no. 6, pp. 585-595, 1999.

[4] S. M. Alamouti, "A simple transmit diversity technique for wireless communications," IEEE Journal on Selected Areas in Communications, vol. 16, no. 8, pp. 1451-1458, 1998.

[5] V. Tarokh, N. Seshadri, and A. R. Calderbank, "Space-time codes for high data rate wireless communication: Performance criterion and code construction," IEEE Transactions on Information Theory, vol. 44, no. 2, pp. 744$765,1998$.

[6] J. Mietzner, R. Schober, L. Lampe, W. H. Gerstacker, and P. A. Hoeher, "Multiple-Antenna techniques for wireless communications: a comprehensive literature survey," IEEE Communications Surveys \& Tutorials, vol. 11, no. 2, pp. 87-105, 2009.

[7] M. Abuthinien, S. Chen, and L. Hanzo, "Semi-blind joint maximum likelihood channel estimation and data detection for MIMO systems," IEEE Signal Processing Letters, vol. 15, pp. 202-205, 2008.

[8] A. Medles and D. T. M. Slock, "Semi-blind channel estimation for MIMO spatial multiplexing systems," in Proceedings of the Vehicular Technology Conference (VTC2001-Fall), Atlantic City, NJ, 2001, pp. 1240-1244.

[9] C. Cozzo and B. L. Hughes, "Joint channel estimation and data detection in space-time communications," IEEE Transactions on Communications, vol. 51, no. 8, pp. 1266-1270, 2003.

[10] A. K. Jagannatham and B. D. Rao, "Whitening-rotation-based semi-blind MIMO channel estimation," IEEE Transactions on Signal Processing, vol. 54, no. 3, pp. 861-869, 2006.

[11] A. K. Jagannatham and B. D. Rao, "A semi-blind technique for MIMO channel matrix estimation," in Proceedings of the IEEE Workshop on Signal Processing Advances in Wireless Communications (SPAWC2003), Rome, Italy, 2003, pp. 304-308.

[12] J. K. Bhalani, D. Chauhan, Y. P. Kosta, and A. I. Trivedi, "Near optimal performance joint semi-blind channel estimation and data detection techniques for Alamouti coded single-carrier (SC) MIMO communication systems," Physical Communication, vol. 8, pp. 31-37, 2013.

[13] F. Wan, W. P. Zhu, and M. N. S. Swamy, "A signal perturbation free whitening-rotation-based semiblind approach for MIMO channel estimation," IEEE Transactions on Signal Processing, vol. 57, no. 8, pp. 3154-3166, 2009.

[14] S. S. Moghaddam and H. Saremi, "A novel semi-blind channel estimation scheme for Rayleigh flat fading MIMO channels (Joint LS estimation and ML detection)," IETE Journal of Research, vol. 56, no. 4, pp. 193-201, 2010.

[15] V. Buchoux, O. Cappe, E. Moulines, and A. Gorokhov, "On the performance of semi-blind subspace-based channel estimation," IEEE Transactions on Signal Processing, vol. 48, no. 6, pp. 1750-1759, 2000. 
[16] S. Zhou, B. Muquet, and G. B. Giannakis, "Sub-space based (semi-) blind channel estimation for block precoded space-time OFDM," IEEE Transactions on Signal Processing, vol. 50, no. 5, pp. 1215-1228, 2002.

[17] A. Medles, D. T. M. Slock, and E. De Carvalho, "Linear prediction based semi-blind estimation of MIMO FIR channels," in Proceedings of the 3rd IEEE Workshop on Signal Processing Advances in Wireless Communications (SPAWC'01), Taiwan, 2001, pp. 58-61.

[18] X. Deng, A. M. Haimovich, and J. Garcia-Frias, "Decision directed iterative channel estimation for MIMO systems," in Proceedings of IEEE International Conference on Communications (ICC'03), Anchorage, AK, 2003, pp. 2326-2329.

[19] O. Edfors, M. Sandell, J. J. van de Beek, S. K. Wilson, and P. O. Borjesson, "OFDM channel estimation by singular value decomposition," IEEE Transactions on Communications, vol. 46, no. 7, pp. 931-939, 1998.

[20] Y. Li, "Pilot-symbol-aided channel estimation for OFDM in wireless systems", IEEE Transactions on Vehicular Technology, vol. 49, no. 4, pp. 1207-1215, 2000.

[21] Y. Li, L. J. Cimini Jr, and N. R. Sollenberger, "Robust channel estimation for OFDM systems with rapid dispersive fading channels," IEEE Transactions on Communications, vol. 46, no. 7, pp. 902-915, 1998.

[22] B. Muquet, M. De Courville, and P. Duhamel, "Subspace-based blind and semi-blind channel estimation for OFDM systems," IEEE Transactions on Signal Processing, vol. 50, no. 7, pp. 1699-1712, 2002.

[23] C. Li and S. Roy, "Subspace-based blind channel estimation for OFDM by exploiting virtual carriers," IEEE Transactions on Wireless Communications, vol. 2, no. 1, pp. 141-150, 2003.

[24] T. Cui and C. Tellambura, "Joint channel estimation and data detection for OFDM systems via sphere decoding," in Proceedings of IEEE Global Telecommunications Conference (GLOBECOM'04), Dallas, TX, 2004, pp. 3656-3660.

[25] T. Cui and C. Tellambura, "Joint data detection and channel estimation for OFDM systems," IEEE Transactions on Communications, vol. 54, no. 4, pp.670-679, 2006.

[26] M. X. Chang and Y. T. Su, "Blind and semi-blind detections of OFDM signals in fading channels," IEEE Transactions on Communications, vol. 52, no. 5, pp.744-754, 2004.

[27] B. Hassibi and H. Vikalo. "On the sphere decoding algorithm. Part I: The expected complexity," IEEE Transactions on Signal Processing, vol. 53, no. 8, pp. 2806-2818, 2005.

[28] Q. Li and Z. Wang. "Improved k-best sphere decoding algorithms for MIMO systems," in Proceedings of IEEE International Symposium on Circuits and Systems (ISCAS2006), Island of Kos, Greece, 2006.

[29] V. Neder, D. Ezri, and M. Haridim, "Low complexity sphere decoding for spatial multiplexing MIMO," 2008; http://arxiv.org/ftp/arxiv/papers/0802/0802.4390.pdf.

[30] J. G. Andrews, A. Ghosh, and R. Muhamed, Fundamentals of WiMAX: Understanding Broadband Wireless Networking. Upper Saddle River, NJ: Prentice Hall, 2007, pp. 149-198.

[31] X. Zhu and R. D. Murch, "Performance analysis of maximum likelihood detection in a MIMO antenna system," IEEE Transactions on Communications, vol. 50, no. 2, pp. 187-191, 2002.

[32] F. Zhao and S. Qiao, "Radius selection algorithms for sphere decoding," in Proceedings of the 2nd Canadian Conference on Computer Science and Software Engineering (C3S2E'09), Montreal, Canada, 2009, pp. 169-174.

[33] G. R. Patil and V. K. Kokate, "Simplified implementation of QRD-M algorithms for MIMO wireless communication system”, CiiT International Journal of Wireless Communication, vol. 5, no. 9, pp. 392-397, 2013.

[34] G. R. Patil and V. K. Kokate, "Simulation study of some spatial multiplexing techniques for MIMO wireless communication systems," International Journal of Electronics Communication and Computer Engineering, vol. 3, no. 6, pp. 1568-1573, 2012.

[35] S. M. Kay, Fundamentals of Statistic Signal Processing: Estimation Theory. Englewood Cliffs, NJ: PTR PrenticeHall, 1993. 


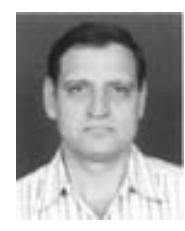

\section{Gajanan R. Patil}

He received the B.Tech. in electronics engineering in 1989 from NIT Calicut, M.E. in Electronics from Govt. College of Engineering, Pune in 1992. He is Pursuing his Ph.D. degree at Sinhgad College of Engineering, Pune. Since 1997 he has been with Department of Electronics \& Telecommunication Engineering at Army Institute of Technology, Pune where he is currently working as Associate Professor. He has published ten text books in the field of Electronics and Communication Engineering. His research interests are Wireless Communications, MIMO Channel Estimation \& detection and Coding. He is member of IEEE and IEEE Comm. Soc.

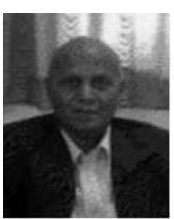

\section{Vishwanath K. Kokate}

He received his B.E. \& M.E. degree in Electronics \& Telecommunication Engineering from Govt. College of Engineering, Pune in 1972 and 1977, respectively. He is currently Ph.D. Guide at Sinhgad College of Engineering, Pune. He has more than 35 years of teaching experience. He was Director, Government College of Engineering, Pune from 2005 to 2007. He has guided more than 10 Ph.D. students. He has worked on number of committees of Government of Maharashtra and AICTE. He has published number of research papers in National, International Conferences and Journals. His research interests include Microwave, antenna and wireless communications. 